\title{
First demonstration of multimode amplifier for spatial division multiplexed transmission systems
}

\author{
Y. Yung, ${ }^{1 *}$ S. Alam, ${ }^{1}$ Z. Li, ${ }^{1}$ A. Dhar, ${ }^{1}$ D. Giles, ${ }^{2}$ I. Giles, ${ }^{2}$ J. Sahu, ${ }^{1}$ L. Grüner-Nielsen, ${ }^{3}$ \\ F. Poletti, ${ }^{1}$ and D. J. Richardson ${ }^{1}$ \\ ${ }^{1}$ Optoelectronics Research Centre, University of Southampton, Southampton, SO17 1BJ, UK \\ ${ }^{2}$ Phoenix Photonics Ltd, 22 Carlton Road, Surrey, CR2 OBS, UK \\ ${ }^{3}$ OFS Fitel Denmark, Priorparken 680, 2605 Brøndby, Denmark \\ *Corresponding author: ymj@orc.soton.ac.uk
}

\begin{abstract}
We present the first systematic study of a two-moded erbium-doped fiber amplifier, and identified its performance trends. By optimizing dopant distribution and pump modes we achieved over $22 \mathrm{~dB}$ gain for both $\mathrm{LP}_{01}$ and $\mathrm{LP}_{11}$-modes.

OCIS codes: (060.0060) Fiber optics and optical communications; (060.2320) Fiber optics amplifiers and oscillators.
\end{abstract}

\section{Introduction}

The exponential growth of internet traffic [1] requires improvements in the existing backbone network technology to increase drastically its transmission capacity. It is envisaged that the information carrying capacity of single-core, single-mode fiber is reaching its fundamental limits and radically new fiber designs and network architectures may soon be needed. To address this issue, spatial division multiplexing (SDM) schemes employing either multi core fibers (MCFs) or multi-mode fibers (MMFs) have been recently proposed and successfully demonstrated in unamplified systems [2,3]. SDM in principle allows upscaling of the capacity per fiber by a factor proportional to the number of cores or modes. However, operation of such schemes in existing long haul networks requires the development of suitable high performance in-line optical amplifiers. Recently a multicore erbium doped fiber (MCEDF) amplifier has been demonstrated with $4 \mathrm{~dB}$ of gain variation between the cores at a net signal gain of $25 \mathrm{~dB}$ [4].

Use of MMF is another attractive option, where each guided mode can in principle be used as an independent channel. Neng et. al. [5] have recently studied numerically the performance of a two-moded amplifier based on a uniformly doped step-index EDF. They have shown that controlling the modal shape of the pump provides a means of tailoring the overall inter-modal gain. In this work we implement and characterize such a two mode erbium doped fiber amplifier. Besides experimentally studying the impact of using different modal pump fields, we also investigate the effect of tailoring the spatial refractive index and $\mathrm{Er}^{3+}$-ion doping concentration in the EDF. Using an optimized active fiber profile we demonstrate over $22 \mathrm{~dB}$ of small signal gain for both $\mathrm{LP}_{01}$ and $\mathrm{LP}_{11}$ modes and a relatively flat gain profile with a maximum gain variation of $3 \mathrm{~dB}$ across the full $\mathrm{C}$-band for $\mathrm{LP}_{01}$. As best we are aware this is the first experimental demonstration of a multimode erbium doped fiber amplifier (MM-EDFA) for SDM transmission.

\section{Experimental results}

The experimental setup of the MM- EDFA is shown in Fig. 1a.
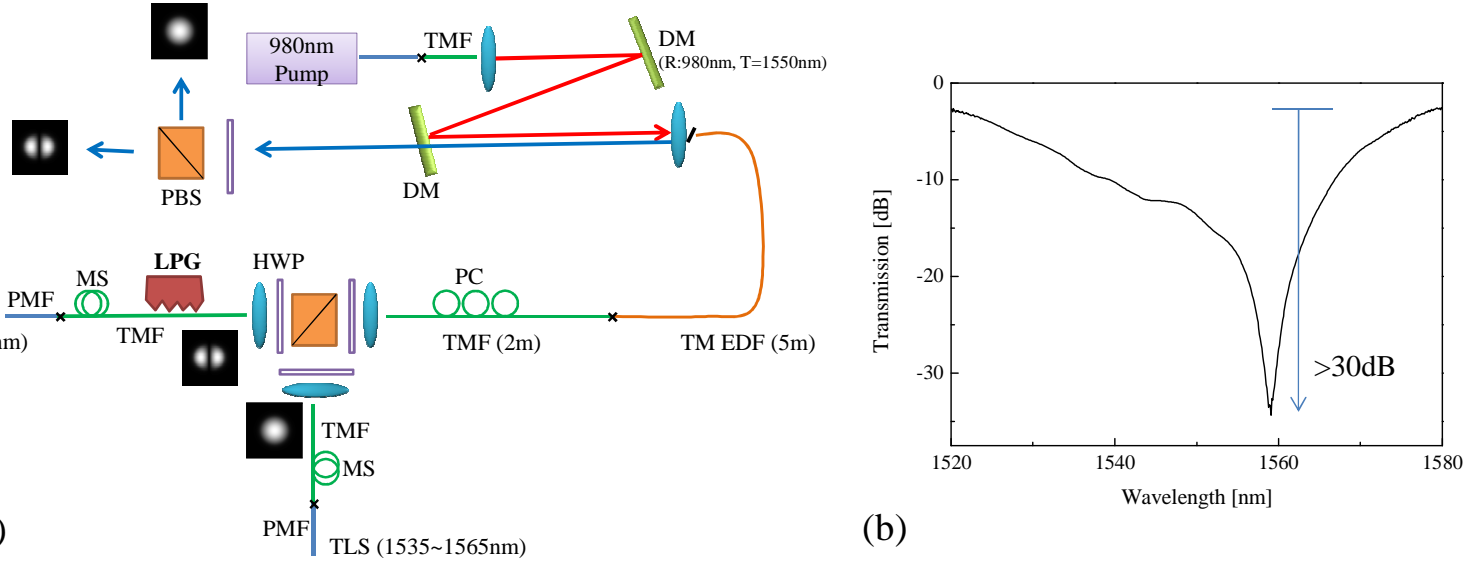

(b)

Fig. 1: (a) Experimental layout to characterize MM-EDFA. LD: Laser Diode, PMF: Polarization Maintaining Fiber, MS: Mode Stripper (LP 11 ), TMF: Two Moded Fiber, LPG: Long Period Grating, HWP: Half Wave Plate, PBS: Polarization Beam Splitter, PC: Polarization Controller, DM: Dichroic Mirror, TM-EDF: Two Moded Erbium Doped Fiber; (b) Typical transmission curve of the mechanical LPG. 
The seed comprised of an external cavity laser (ECL) tunable across the C-band and a fixed wavelength DFB diode laser at $1558.9 \mathrm{~nm}$. The single-mode, single polarized output of the ECL and the DFB were spliced to passive multimode optical fibers. For simplicity we used a fiber with a core diameter of $19.7 \mu \mathrm{m}$ and a numerical aperture (NA) of 0.12 which effectively guides only two transverse mode groups, namely the $\mathrm{LP}_{01}$ and $\mathrm{LP}_{11}$ mode groups ( comprising 6 distinct modes including all degeneracies and polarizations). A mechanical long period grating (LPG) was applied to the two moded fiber (TMF) spliced to the end of the DFB pigtail to convert input light in the $\mathrm{LP}_{01}$ to the $\mathrm{LP}_{11}$ mode. A typical transmission curve of the mechanical LPG is shown in Fig. 1b showing that conversion to the $\mathrm{LP}_{11}$ mode was achieved with an extinction ratio of $30 \mathrm{~dB}$ when a single polarization $\mathrm{LP}_{01}$ mode at $1559 \mathrm{~nm}$ was coupled into the TMF. For ease of demultiplexing the modes at the amplifier output we considered only the $\mathrm{x}$ polarized $\mathrm{LP}_{01}$ mode (i.e. $\mathrm{LP}^{\mathrm{x}}{ }_{01}$ ) and the $\mathrm{LP}_{11}$ mode along the y-polarization, i.e. $\mathrm{LP}_{11}^{\mathrm{y}}$. The $\mathrm{LP}^{\mathrm{x}}{ }_{01}$ mode from the ECL and $\mathrm{LP}^{\mathrm{y}}{ }_{11}$ from the DFB are polarization multiplexed using a polarization beam splitter (PBS) and coupled into a second length of passive TMF. The launch end of the TMF was flat cleaved to enable clean excitation of the fiber modes (this has the slight downside that it leads to an increased buildup of amplified spontaneous emission (ASE) at the amplifier output at low input signal powers). The output of the input length of passive TMF was analyzed to assess the degradation in polarization extinction ratio (PER) between the two orthogonal modes as well as any mode cross-coupling during the free space coupling. A PER in excess of $20 \mathrm{~dB}$ was measured for both the modes, which is sufficient for amplification and polarization demultiplexing of the two orthogonal signal modes at the output of the amplifier. The passive fiber was then spliced directly to a $5 \mathrm{~m}$ length of either of two mode-matched $\mathrm{Er}^{3+}$-doped active fibers each exhibiting different fiber refractive index profiles (FRIPs), namely Fiber \#1 and Fiber \#2, as shown in Fig. $2 \mathrm{a}$ and Fig. $2 \mathrm{~b}$ respectively. It is reasonable to assume that the $\mathrm{Er}^{3+}$-doping profile follows the refractive index profiles of the active fibers. A $980 \mathrm{~nm}$ fiber pigtailed diode laser was used to pump the active fibers and was free-space coupled into the MM-EDFA using a dichroic mirror. The free ends of the active fibers were angle cleaved to suppress Fresnel reflection. A second PBS was used at the output of the amplifier to separate the two orthogonal polarization modes.

(a)

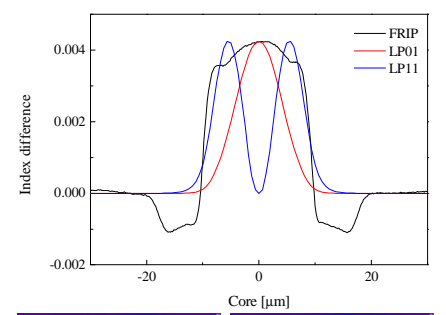

(c)

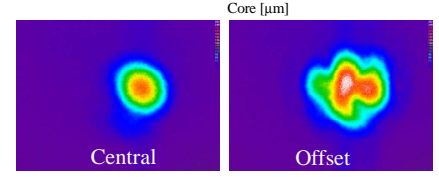

(e)

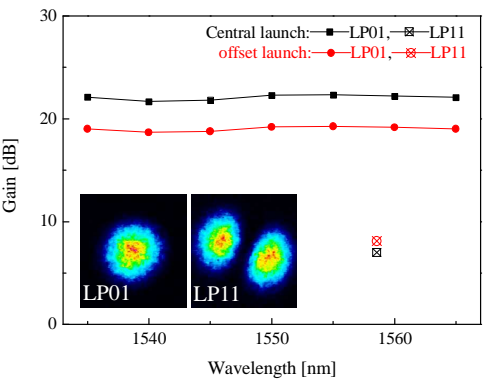

(b)

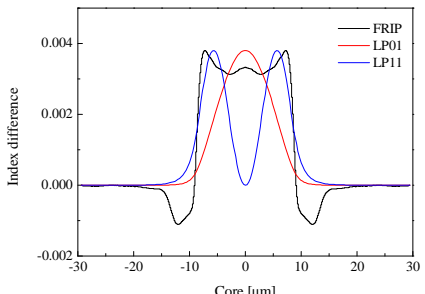

(d)

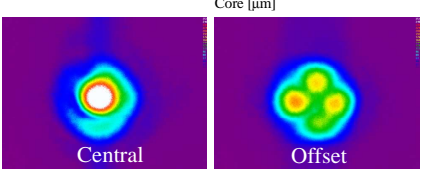

(f)

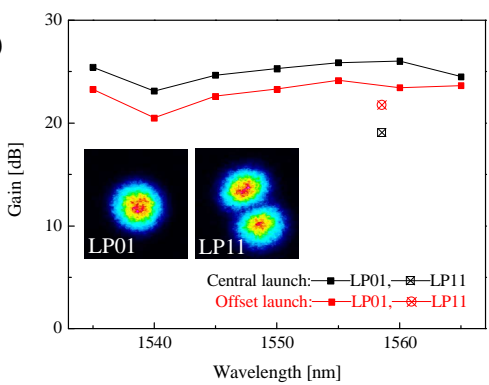

Fig. 2: (a, b) FRIP and calculated mode profiles supported by the two fabricated active fibers, (c, d) pump mode profiles coupled into the respective doped fibers for center and offset launch, $(\mathrm{e}, \mathrm{f})$ gain vs wavelength for $\mathrm{LP}^{\mathrm{x}}{ }_{01}$ and $\mathrm{LP}^{\mathrm{y}}{ }_{11}$ modes for center and offset launch.

The amplified output powers of the $\mathrm{LP}^{\mathrm{x}}{ }_{01}$ and $\mathrm{LP}^{\mathrm{y}}{ }_{11}$ modes, shown in Fig. 2e and $2 \mathrm{f}$, critically depend on the pump modes. For centered launch condition which promotes the excitation of the symmetric pump modes (Fig. 2c and Fig. 2d), the measured gain of the $\mathrm{LP}^{\mathrm{x}}{ }_{01}$ mode for Fiber \#1 (Fig. 2a) was found to be substantially higher $(>15 \mathrm{~dB})$ than the $\mathrm{LP}_{11}^{\mathrm{y}}$ mode due to better spatial overlap with the pump modes as well as the $\mathrm{Er}^{3+}$-dopant distribution. For offset launch the asymmetric pump modes dominate over the symmetric ones and the differential gains of the two modes were measured to be around 10dB. However this comes at the expense of lower average output power due the reduced pump coupling efficiency. Fiber \#2 was designed to have higher $\mathrm{Er}^{3+}-$ density at the 
edges of the refractive index profile so as to have reduced pump field sensitivity to the modal gains. For centered launch condition the gain difference between the $\mathrm{LP}^{\mathrm{x}}{ }_{01}$ and $\mathrm{LP}_{11}^{\mathrm{y}}$ mode was measured to be $\sim 6 \mathrm{~dB}$ for an input signal power of $-10 \mathrm{dBm}$. This could be brought down to within $1 \mathrm{~dB}$ with the optimum offset launch shown in Fig. $2 \mathrm{~d}$. These results show that the differential gain between the two signal modes can be managed by controlling either the relative strength of the pump modes as indicated in Ref [6], or the $\mathrm{Er}^{3+}$-doping distribution, or both. Large scale gain differential between the modes can be addressed by manipulating the FRIP whilst fine tuning can be achieved by controlling the strength of the individual pump modes. Since Fiber \#2 achieves a lower differential gain between the two modes, we will focus our attention on this fiber only for more detailed investigation of the MM-EDFA.

Fig. 3 shows the amplifier gain as a function of input signal power for centered and offset launch conditions when the wavelengths of the $\mathrm{LP}^{\mathrm{x}}{ }_{01}$ and $\mathrm{LP}^{\mathrm{y}}{ }_{11}$ modes are either separated by $\sim 9 \mathrm{~nm}$ or overlap with each other (i.e. degenerate signal wavelength). To minimize uncertainly in the pump launch condition, the single-mode pigtail of the diode laser was first spliced to a section of the passive TMF with no offset to simulate the centered launch condition and then with a slight offset between the cores to simulate the offset launch condition such that the $\mathrm{LP}_{21}$ pump mode was observed at the output of the TMF. The output of the TMF in both cases was then collimated and focused into the TMF-EDF. For centered launch, the gain difference of the $\mathrm{LP}^{\mathrm{x}}{ }_{01}$ over the $\mathrm{LP}^{\mathrm{y}}{ }_{11}$ mode decreased from $\sim 10 \mathrm{~dB}$ to $\sim 5 \mathrm{~dB}$ as the input signal power was increased from $-15 \mathrm{dBm}$ to $-5 \mathrm{dBm}$. Notably the $\mathrm{LP}^{\mathrm{x}}{ }_{01}$ mode experienced gain compression from a very low signal input power whilst the gain of the LP $^{\mathrm{y}}{ }_{11}$ mode saturates to a value of $17 \mathrm{~dB}$ as the input power increased to $-10 \mathrm{dBm}$ and beyond. For offset launch however the gain difference decreased markedly at all input powers and reduced to within $1 \mathrm{~dB}$ at an input power of $-5 \mathrm{dBm}$. Both modes experienced gain reduction with an increase in input signal powers. The amplifier behaved similarly when the wavelengths of the $\operatorname{LP}_{01}^{\mathrm{x}}$ and $\mathrm{LP}^{\mathrm{y}}{ }_{11}$ modes were set to $1558.9 \mathrm{~nm}$. The CCD images of the two modes as shown in Fig. $3 \mathrm{~b}$ are well defined at high signal input powers however the quality of the images degraded with decreasing input signal power due to the increasing dominance of unpolarized ASE light in both the transverse modes and which appears in both arms after the PBS. A maximum output power of $82 \mathrm{~mW}$ was measured at an optical-to-optical conversion efficiency of $\sim 32 \%$.

(a)

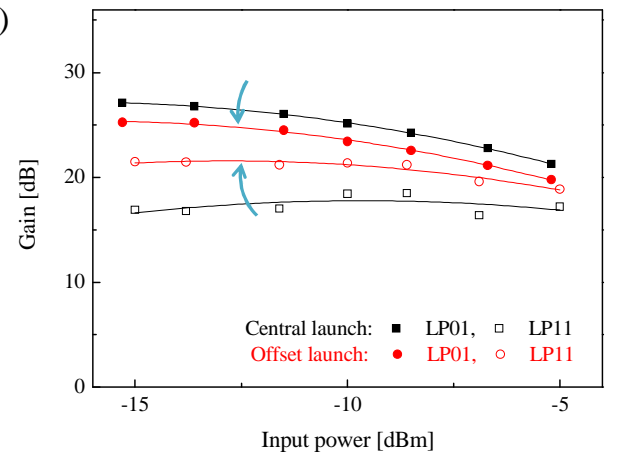

(b)

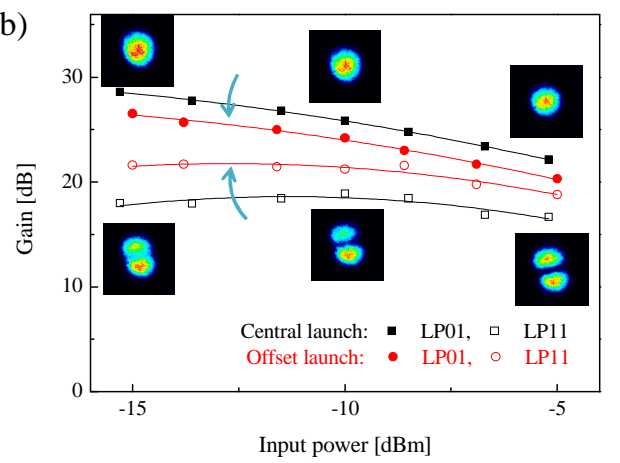

Fig. 3: Gain as a function of input power for centered and offset launch when signal wavelengths are (a) $9 \mathrm{~nm}$ apart and (b) overlapped.

\section{Conclusions}

We present the first implementation of a MM-EDFA for SDM transmission. We demonstrate that differential modal gain can be significantly improved by optimizing the pump launch and the input signal power. We also propose an improved EDF with a tailored central dip in both the refractive index and Er-ion concentration, and show its advantages over a more standard fiber profile. Using this fiber with an offset pump launch exciting prevalently the $\mathrm{LP}_{21}$ mode we achieved over $22 \mathrm{~dB}$ gain for two modes at the same wavelength and in orthogonal polarizations. Characterization of the MM-EDFA with multiple modes in the same polarization and for a larger number of input modes is currently ongoing. This work was supported by the European Communities 7th Framework Programme under grant agreement 228033 (MODE-GAP).

\section{References}

[1] A. Ellis et al., “Approaching the Non-Linear Shannon Limit,” J. Lightwave Technol. 28, 423-433 (2010)

[2] R. Ryf et al., "Space-division multiplexing over 10km of three-mode fiber using coherent 6x6 MIMO processing," in Proc. of OFC/NFOEC' 11 (Optical Society of America, Washington, 2011), PDPB10.

[3] Jun Sakaguchi et al., "109-Tb's (7x97x1720Gb/s SDM/WDM/PDM) QPSK transmission through 16.8-km homogeneous multi-core fiber," in Proc. of OFC/NFOEC'11 (Optical Society of America, Washington, 2011), PDPB6.

[4] K. S. Abedin, T. F. Taunay, M. Fishteyn, M. F. Yan, B. Zhu, J. M. Fini, E. M. Monberg, F. V. Dimarcello and P. W. Wisk, "Amplification and noise properties of an erbium-doped multicore fiber amplifier", Opt. Exp. 19, 16715-16721 (2011).

[5] N. Bai, E. Ip, T. Wang and G. Li, "Multimode fiber amplifier with tunable modal gain using a reconfigurable multimode pump", Opt. Exp. 19, 16601-16611 (2011). 